\title{
ON IMPLICIT HEAVY SUBGRAPHS AND HAMILTONICITY OF 2-CONNECTED GRAPHS
}

\author{
Wei Zheng ${ }^{a}$, Wojciech Widę $^{b}$ \\ AND \\ LIGONG WANG ${ }^{a, 1}$ \\ ${ }^{a}$ Department of Applied Mathematics, School of Science \\ Northwestern Polytechnical University \\ Xi'an, Shaanxi 710072, P.R. China \\ ${ }^{b}$ Univ Rennes, INSA Rennes, CNRS, IRISA, Rennes, France \\ e-mail: zhengweimath@163.com \\ wwidel@irisa.fr \\ lgwangmath@163.com
}

\begin{abstract}
A graph $G$ of order $n$ is implicit claw-heavy if in every induced copy of $K_{1,3}$ in $G$ there are two non-adjacent vertices with sum of their implicit degrees at least $n$. We study various implicit degree conditions (including, but not limiting to, Ore- and Fan-type conditions) imposing of which on specific induced subgraphs of a 2-connected implicit claw-heavy graph ensures its Hamiltonicity. In particular, we improve a recent result of [X. Huang, Implicit degree condition for Hamiltonicity of 2-heavy graphs, Discrete Appl. Math. 219 (2017) 126-131] and complete the characterizations of pairs of o-heavy and f-heavy subgraphs for Hamiltonicity of 2-connected graphs.
\end{abstract}

Keywords: implicit degree, implicit o-heavy, implicit f-heavy, implicit cheavy, Hamilton cycle.

2010 Mathematics Subject Classification: 05C45, 05C38, 05 C07.

\section{REFERENCES}

[1] P. Bedrossian, Forbidden Subgraph and Minimum Degree Conditions for Hamiltonicity, Ph.D. Thesis (Memphis State University, USA, 1991).

\footnotetext{
${ }^{1}$ Corresponding author.
} 
[2] P. Bedrossian, G. Chen and R.H. Schelp, A generalization of Fan's condition for Hamiltonicity, pancyclicity and Hamiltonian connectedness, Discrete Math. 115 (1993) 39-50. doi:10.1016/0012-365X(93)90476-A

[3] J.A. Bondy and U.S.R. Murty, Graph Theory with Applications, GTM 244 (Springer, Berlin, 2008).

[4] H.J. Broersma, Z. Ryjáček and I. Schiermeyer, Dirac's minimum condition restricted to claws, Discrete Math. 167/168 (1997) 155-166. doi:10.1016/S0012-365X(96)00224-5

[5] H.J. Broersma and H.J. Veldman, Restrictions on induced subgraphs ensuring Hamiltonicity or pancyclicity of $K_{1,3}$-free graphs, in: Contemporary Methods in Graph Theory, (BI Wissenschaftsverlag, Mannheim, 1990) 181-194.

[6] J. Brousek, Forbidden triples for Hamiltonicity, Discrete Math. 251 (2002) 71-76. doi:10.1016/S0012-365X(01)00326-0

[7] R. Čada, Degree conditions on induced claws, Discrete Math. 308 (2008) 5622-5631. doi:10.1016/j.disc.2007.10.026

[8] J. Cai and H. Li, Hamilton cycles in implicit 2-heavy graphs, Graphs Combin. 32 (2016) 1329-1337. doi:10.1007/s00373-015-1669-4

[9] J. Cai and Y. Zhang, Fan-type implicit-heavy subgraphs for Hamiltonicity of implicit claw-heavy graphs, Inform. Process. Lett. 116 (2016) 668-673. doi:10.1016/j.ipl.2016.06.012

[10] G. Chen, B. Wei and X. Zhang, Forbidden graphs and Hamiltonian cycles, preprint, 1995.

[11] G. Chen, B. Wei and X. Zhang, Degree-light-free graphs and Hamiltonian cycles, Graphs Combin. 17 (2001) 409-434. doi: $10.1007 / \mathrm{s} 003730170018$

[12] B. Chen and S. Zhang, An implicit degree condition for long cycles in 2-connected graphs, Appl. Math. Lett. 19 (2006) 1148-1151. doi:10.1016/j.aml.2005.12.006

[13] V. Chvátal and P. Erdős, A note on Hamiltonian circuits, Discrete Math. 2 (1972) 111-113. doi:10.1016/0012-365X(72)90079-9

[14] D. Duffus, R.J. Gould and M.S. Jacobson, Forbidden subgraphs and the Hamiltonian theme, Proceedings of the 4th International Conference on the Theory and Applications of Graphs, Kalamazoo, 1980 (Wiley, New York, 1981) 297-316.

[15] G. Fan, New sufficient conditions for cycles in graphs, J. Combin. Theory Ser. B 37 (1984) 221-227. doi:10.1016/0095-8956(84)90054-6 
[16] R. Faudree, Z. Ryjáček and I. Schiermeyer, Forbidden subgraphs and cycle extendability, J. Combin. Math. Combin. Comput. 19 (1995) 109-128.

[17] R.J. Faudree and R.J. Gould, Characterizing forbidden pairs for Hamiltonian properties, Discrete Math. 173 (1997) 45-60. doi:10.1016/S0012-365X(96)00147-1

[18] S. Goodman and S.T. Hedetniemi, Sufficient conditions for a graph to be Hamiltonian, J. Combin. Theory Ser. B 16 (1974) 175-180. doi:10.1016/0095-8956(74)90061-6

[19] R.J. Gould and M.S. Jacobson, Forbidden subgraphs and Hamiltonian properties of graphs, Discrete Math. 42 (1982) 189-196. doi:10.1016/0012-365X(82)90216-3

[20] Z. Hu, A generalization of fan's condition and forbidden subgraph conditions for Hamiltonicity, Discrete Math. 196 (1999) 167-175. doi:10.1016/S0012-365X(98)00200-3

[21] X. Huang, Hamilton cycles in implicit claw-heavy graphs, Inform. Process. Lett. 114 (2014) 676-679. doi:10.1016/j.ipl.2014.06.007

[22] X. Huang, Implicit degree condition for Hamiltonicity of 2-heavy graphs, Discrete Appl. Math. 219 (2017) 126-131. doi:10.1016/j.dam.2016.10.031

[23] B. Li and B. Ning, Heavy subgraphs, stability and Hamiltonicity, Discuss. Math. Graph Theory 37 (2017) 691-710. doi:10.7151/dmgt.1967

[24] B. Li, B. Ning and S. Zhang, Degree conditions restricted to induced paths for Hamiltonicity of claw-heavy graphs, Acta Math. Sin. (Engl. Ser.) 33 (2017) 301-310. doi:10.1007/s10114-016-5735-5

[25] B. Li, Z. Ryjáček, Y. Wang and S. Zhang, Pairs of heavy subgraphs for Hamiltonicity of 2-connected graphs, SIAM J. Discrete Math. 26 (2012) 1088-1103. doi:10.1137/11084786X

[26] B. Li and P. Vrána, Forbidden pairs of disconnected graphs implying Hamiltonicity, J. Graph Theory 84 (2017) 249-261. doi:10.1002/jgt.22024

[27] G. Li, B. Wei and T. Gao, A structural method for Hamiltonian graphs, Australas. J. Combin. 11 (1995) 257-262.

[28] H. Li, W. Ning and J. Cai, An implicit degree condition for cyclability in graphs, Lecture Notes in Comput. Sci. 6681 (2011) 82-89. doi:10.1007/978-3-642-21204-8_12

[29] B. Ning, Fan-type degree condition restricted to triples of induced subgraphs ensuring Hamiltonicity, Inform. Process. Lett. 113 (2013) 823-826. doi:10.1016/j.ipl.2013.07.014 
[30] B. Ning and S. Zhang, Ore- and Fan-type heavy subgraphs for Hamiltonicity of 2connected graphs, Discrete Math. 313 (2013) 1715-1725.

doi:10.1016/j.disc.2013.04.023

[31] O. Ore, Note on Hamilton circuits, Amer. Math. Monthly 67 (1960) 55. doi: $10.2307 / 2308928$

[32] Y. Zhu, H. Li and X. Deng, Implicit-degrees and circumferences, Graphs Combin. 5 (1989) 283-290.

doi:10.1007/BF01788680

Received 29 May 2017

Revised 20 August 2018

Accepted 29 August 2018 